\title{
Flavonoid Compounds Are Enriched in Lemon Balm (Melissa officinalis) Leaves by a High Level of Sucrose and Confer Increased Antioxidant Activity
}

\author{
Md. Aktar Hossain, Sooah Kim, Kyoung Heon Kim, Sung-Joon Lee, \\ and Hojoung Lee ${ }^{1}$ \\ College of Life Sciences and Biotechnology, Korea University, Anam-Dong, \\ Sungbuk-ku, Seoul 136-713, Republic of South Korea
}

Additional index words. anthocyanins, antioxidant activity, flavonoid, Melissa officinalis, phytohormones, principal components, sucrose concentrations

\begin{abstract}
Medicinal plants are widely used in traditional medicine because plant secondary metabolites have been shown to benefit a broad spectrum of health conditions. Lemon balm, Melissa officinalis L., a member of the mint family, is native to Europe and is well known for its ability to reduce stress and anxiety, promote sleep, and ease pain and discomfort associated with digestion. In various plant species, strong anthocyanin induction is triggered by sucrose, but not by other sugars or osmotic stress; however, the mechanisms that induce anthocyanin accumulation in lemon balm leaves in response to sucrose and phytohormones remain unclear. In this study, we investigated the mechanisms that lead to increased levels of flavonoids in lemon balm plants. We observed that sucrose significantly increases the level of flavonoids in lemon balm plants and that sucrose induction appears to be mediated by the phytohormones abscisic acid and ethylene. We also identified delphinidin as the anthocyanidin that is primarily enriched in leaves grown in high-sucrose medium. Finally, we observed that reactive oxygen species levels are positively correlated with sucrose-mediated anthocyanin accumulation. Taken together, our results demonstrate that the level of flavonoids in lemon balm can be increased significantly and that plants such as lemon balm could potentially be used to prevent diseases that have been purported to be caused by free radical damage. Chemical abbreviations used: ABA, (+)-cis, transabscissic acid; ACC, 1-aminocyclopropanecarboxylic acid; $\mathrm{CHI}$, chalcone isomerase; $\mathrm{CHS}$, chalcone synthase; DPPH, 2, 2diphenyl-1-picrylhydrazyl; GA, gibberellic acid; IAA, indole-3-acetic acid.
\end{abstract}

More than 30,000 different secondary metabolites are produced exclusively by plants. One such class of compounds, the flavonoids (polyphenolic compounds), are classified according to their chemical structures into flavonols, flavones, flavanones, isoflavones, catechins, anthocyanidins, and chalcones. These metabolites have a broad spectrum of action in plants; for example, they protect against pathogen attack, act as attractants for pollinators, and have been used as colorants, as scents, and for allelopathy. Flavonoids also contain chemical structural elements that are responsible for antioxidation, and their antioxidant activities have been well established biochemically.

Lemon balm, Melissa officinalis, is a member of the mint family that is native to Europe. Its use as a medicinal herb dates from the Middle Ages, and it is very well

Received for publication 13 July 2009. Accepted for publication 28 Sept. 2009.

This work was supported by a grant from ARPC (to H.L., Grant \# 107100-03-1-SB010) and in part by a grant from ARPC (to H.L., Grant \# 10806603-1-HD120).

${ }^{1}$ To whom reprint requests should be addressed; e-mail lhojoung@korea.ac.kr. known for its ability to reduce stress and anxiety, promote sleep, improve appetite, and ease pain and discomfort associated with digestion. Moreover, several studies suggest that lemon balm is beneficial for a wide variety of human disorders such as cancer, HIV-1, Alzheimer's disease, attention deficit hyperactivity disorder, indigestion, gas, insomnia, and hyperthyroidism (de Sousa et al., 2004; Galasinski et al., 1996; Geuenich et al., 2008; Kennedy et al., 2004, 2006; Muller and Klement, 2006; Yamasaki et al., 1998).

Because plants are autotrophic organisms, they need to synthesize sugars for growth and storage. Sugars appear to act as hormone-like signaling molecules in plant cells because they can regulate plant metabolism, growth, and development (Rolland et al., 2002; Rook and Bevan, 2003; Smeekens, 2000). For example, in grape skins, sugars induce most of the genes involved in anthocyanin synthesis (Gollop et al., 2002). Recently, sucrose-specific induction of anthocyanin biosynthesis was also examined in Arabidopsis (Solfanelli et al., 2006; Teng et al., 2005), Torenia (Nagira et al., 2006), and Camptotheca (Pasqua et al., 2005). Exogenous growth regulators, especially abscisic acid (ABA), were also reported to enhance antho- cyanin accumulation in Arabidopsis (Loreti et al., 2008) and Torenia (Nagira et al., 2006) when plants were supplied with sucrose. Moreover, several studies have shown that phytohormones are involved in the sucroseregulated expression of genes encoding anthocyanin biosynthetic enzymes in Arabidopsis seedlings (Chen et al., 2007; Devoto et al., 2005; Loreti et al., 2008; Tonelli et al., 2007). However, the mechanisms of anthocyanin accumulation remain unclear in the leaves of lemon balm. Therefore, in this study, we intended to understand the role of sucrose and phytohormones leading to the increased level of anthocyanins in this plant.

\section{Materials and Methods}

Plant growth conditions and chemicals. Explants from containerized lemon balm (Melissa officinalis L.; Lamiaceae) plants grown in sterile Murashige and Skoog (MS) medium $\left(4 \mathrm{~g} \cdot \mathrm{L}^{-1} \mathrm{MS}, 50 \mathrm{~mm}\right.$ sucrose solidified with $7 \mathrm{~g} \cdot \mathrm{L}^{-1}$ agar at $\mathrm{pH}$ 5.7) were used for this study. Explants $\approx 2 \mathrm{~cm}$ in length, including the tip and one pair of leaves, were transferred to the culture/test media and incubated in a controlled environment with a 15-h light/9-h dark regime. To examine the effects of sucrose concentration on plant growth, anthocyanin accumulation, flavonoid biosynthesis, chlorophyll content, and antioxidant activity, explants were grown in MS agar medium for $20 \mathrm{~d}$. To examine the effects of plant growth regulators on anthocyanin production, the explants were grown in MS agar medium containing $50 \mathrm{~mm}$ sucrose for $12 \mathrm{~d}$ and then were transferred to liquid MS medium containing 50 or $300 \mathrm{~mm}$ sucrose. This medium was supplemented with various concentrations of growth regulators. After transfer, the explants were incubated for a further $3 \mathrm{~d}$ before analysis.

All of the chemicals used in this study were purchased from Sigma Aldrich (St. Louis, MO) except for the ABA $[(+)$-cis, transabscissic acid], ACC, indole-3-acetic acid (IAA), fluridon, and silver nitrate, which were purchased from Duchefa Biochemie (Postbus, Haarlem, The Netherlands), and the methanol and ethanol, which were purchased from Merck (Darmstadt, Germany).

Quantification of anthocyanins and chlorophyll content. Anthocyanins were extracted as described by Neff and Chory (1998) with minor modifications. Briefly, $100 \mathrm{mg}$ of fresh leaf samples were ground in liquid nitrogen, added to $250 \mu \mathrm{L}$ of $1 \%$ $\mathrm{HCl}(\mathrm{v} / \mathrm{v})$ in methanol, and extracted in the dark at $4{ }^{\circ} \mathrm{C}$ overnight. Distilled water (250 $\mu \mathrm{L})$ was added to each tube. Extracts were recovered, and the chlorophyll was removed by adding $500 \mu \mathrm{L}$ chloroform, mixing the samples, and centrifuging at $3000 \mathrm{rpm}$ for 2 $\min$. The aqueous phase $(200 \mu \mathrm{L})$ from each sample was then placed in a well of a 96-well plate and the absorbance was determined using a microplate spectrophotometer at $530 \mathrm{~nm}$. The chlorophyll content was measured as described by Porra et al. (1989) with slight modifications. Briefly, two leaf discs 
$\left(0.5 \mathrm{~cm}^{2}\right.$ each) were weighed and placed into $1 \mathrm{~mL}$ of dimethylformamide (DMF) before extraction in the dark (at $4{ }^{\circ} \mathrm{C}$ ) for $24 \mathrm{~h}$. Absorbances were determined against a blank (1 mL DMF) at $647 \mathrm{~nm}$ and $664 \mathrm{~nm}$ using a spectrophotometer.

Liquid chromatography-mass spectrometry analysis. Leaves of lemon balm were ground and homogenized in liquid nitrogen using a mortar and pestle. Sample preparation and extraction were carried out as described by Tolstikov et al. (2007). Methanol (1 mL) was added to $100 \mathrm{mg}$ of the ground sample in a microcentrifuge tube and mixed by vortexing. Reserpine dissolved in methanol at 0.2 $\mathrm{mg} \cdot \mathrm{ml}^{-1}(50 \mu \mathrm{L})$ was added as an internal reference to the sample tube with $50 \mu \Lambda$ of water, and the tube was vortexed for $5 \mathrm{~min}$. The sample was then centrifuged for $5 \mathrm{~min}$ at $14,000 \mathrm{~g}$, and the supernatant was transferred to a glass sample vial (2 $\mathrm{mL}$; Agilent, Wilmington, DE) with a screw cap lined with Teflon.

Sample analysis was conducted using a high-performance liquid chromatography (HPLC) Surveyor MS Pump Plus (ThermoFisher Scientific, San Jose, CA) coupled to an LTQ Orbitrap mass spectrometer (MS) with an electrospray ionization (ESI) source (ThermoFisher Scientific). The compounds were separated using a BetaBasic-18 HPLC column $(150 \times 2.1 \mathrm{~mm}$ i.d., $5 \mu \mathrm{m}$; ThermoFisher Scientific) with aqueous $0.2 \%(\mathrm{v} / \mathrm{v})$ formic acid as solvent $\mathrm{A}$ and $1 \%(\mathrm{v} / \mathrm{v})$ formic acid in methanol as solvent $\mathrm{B}$. The solvent gradient was programmed in the following sequence: $0 \mathrm{~min}, 80 \%$ solvent $\mathrm{A}$; $40 \mathrm{~min}$, $20 \%$ solvent A; $45 \mathrm{~min}, 80 \%$ solvent $\mathrm{A}$; and $60 \mathrm{~min}, 80 \%$ solvent $\mathrm{A}$. The flow rate was 0.2 $\mathrm{mL} \cdot \mathrm{min}^{-1}$, and the sample injection volume was $20 \mu \mathrm{L}$. ESI conditions were as follows: spray voltage of $4.2 \mathrm{kV}$, capillary temperature of $200{ }^{\circ} \mathrm{C}$, capillary voltage of 240 , sheath gas flow rate of 20 arb with nitrogen, aux gas flow rate of 12 arb with nitrogen, and sweep gas flow rate of 10 arb with nitrogen. MS data were acquired over an $\mathrm{m} / \mathrm{z}$ range of 230 to 1200 . The resolution of the mass spectra was set to 60,000 to measure the masses of the compounds.

Data sets organized in matrix form were subsequently exported to SIMCA-P software (Version 11.5; Umetrics, Umea, Sweden) for principal component analysis (PCA). PCA is an unsupervised clustering method that is able to retain the maximum number of variations present in a data set consisting of a large number of interrelated variables while reducing the dimensionality of the data set. Therefore, it is possible to observe any groupings of the data set in a score plot. Coefficients multiplied with the original variables to obtain the principal components (PCs) are called "loadings," and the numerical value of a loading for a given variable on a PC indicates the relationship of the variable with that component (Ma et al., 2007).

Determination of flavonoid content and free radical scavenging activity. Samples for determining the flavonoid content and antioxidant activity of lemon balm leaves were prepared as described by Faudale et al. (2008) with slight modifications. Briefly, $100 \mathrm{mg}$ of fresh leaf samples from each treatment were ground in liquid nitrogen and extracted with $10 \mathrm{~mL}$ of water/ethanol $(80: 20 \mathrm{v} / \mathrm{v})$ three times in $20 \mathrm{~min}$ with sonication. After centrifuging at $7600 \mathrm{~g}$ for $10 \mathrm{~min}$, the supernatant was adjusted to a final volume of $10 \mathrm{~mL}$ and was filtered with a $0.45-\mathrm{nm}$ filter. Extracted leaf samples were quantified immediately after extraction to avoid possible degradation.

The flavonoid contents of lemon balm leaves grown in various concentrations of sucrose were determined using a colorimetric method as described by Faudale et al. (2008) with minor modifications. Briefly, $0.1 \mathrm{~mL}$ of phytochemical extract was diluted with 0.5 $\mathrm{mL}$ of distilled water. Thirty microliters of a $5 \% \mathrm{NaNO}_{2}$ solution were added and the solution was thoroughly mixed by inverting. Six minutes later, $60 \mu \mathrm{L}$ of a $10 \%$ $\mathrm{AlCl}_{3} \cdot 6 \mathrm{H}_{2} \mathrm{O}$ solution was added and the mixture was left to stand for $5 \mathrm{~min}$. Two hundred microliters of $1 \mathrm{M} \mathrm{NaOH}$ were added, and the total volume was brought up to $1 \mathrm{~mL}$ with distilled water. After mixing the solution thoroughly, the absorbance was measured immediately against a prepared blank at $510 \mathrm{~nm}$ using a microplate spectrophotometer. The flavonoid content was calculated using the standard equation $\mathrm{Y}=$ $0.006 \mathrm{X}+0.003\left(R^{2}=0.999\right)$, which was derived from a standard curve generated with $2,4,10$, and $20 \mu \mathrm{g} \cdot \mathrm{mL}^{-1}$ quercetin.

The radical scavenging activities of lemon balm leaves grown in various concentrations of sucrose were determined using the stable radical 2, 2-diphenyl-1-picrylhydrazyl (DPPH) (Brand-Williams et al., 1995) as described by Faudale et al. (2008). Briefly, each leaf extract was diluted to various concentrations ranging from 10 to $200 \mu \mathrm{L} \cdot \mathrm{mL}^{-1}$ in $0.5 \mathrm{ml}$ (methanol for control) and each sample was added to $1.0 \mathrm{~mL}$ of $20 \mathrm{mg} \cdot \mathrm{L}^{-1}$ DPPH dissolved in methanol. After $20 \mathrm{~min}$, the absorbance was measured at $517 \mathrm{~nm}$ with a microplate spectrophotometer. The percentage of DPPH in the sample was calculated according to the following equation: $\%$ decolorization $=[1-($ Abs sample/Abs control $)] \times$ 100. Decoloration was plotted against the sample extract concentrations, and a logarithmic regression curve was established (using GraphPad Prism 5 software; GraphPad Software, Inc., La Jolla, CA) to calculate the $\mathrm{IC}_{50}$, which is the amount of sample necessary to decrease the absorbance of DPPH by $50 \%$.

Reactive oxygen species $\left(\mathrm{H}_{2} \mathrm{O}_{2}\right)$ staining. Young leaves of lemon balm from each treatment were washed with $50 \mu \mathrm{M}$ PBT (potassium phosphate) buffer in a 24-well plate on a rotary shaker for $10 \mathrm{~min}$. The PBT was replaced with $3,3^{\prime}$-diaminobenzidine (DAB) solution in PBT $\left(0.3 \mathrm{mg} \cdot \mathrm{mL}^{-1}\right)$, and samples were incubated for $10 \mathrm{~min}$ at room temperature. For the color reaction, $1 \mu \mathrm{L}$ of $30 \% \mathrm{H}_{2} \mathrm{O}_{2}$ (1:1 in PBT) was added to each leaf, and reactions were shaken vigorously for $10 \mathrm{~min}$. Then, the DAB was replaced with PBT to stop the reaction. The leaves were washed twice in PBT for $5 \mathrm{~min}$ and dehydrated in $100 \%$ methanol overnight for imaging (Fester and Hause, 2005). Stained leaves were stretched on water in six-well plates (Corning Inc., New York, NY), and images were captured with a digital still camera (Sony Corp., Tokyo, Japan).

Statistical analysis. Each experiment was repeated at least three times to confirm reproducibility. All data were statistically analyzed using the SPSS program (Version 13.00; SPSS Inc., Chicago, IL). Analysis of variance and Duncan's multiple range test were performed to assess the possible significant differences among the treatments at the $P \leq 0.05$ level.

\section{Results}

Sucrose dramatically increases anthocyanin levels in lemon balm leaves. There is little information in the literature about optimal growth conditions for cultured lemon balm plants. Therefore, we used trial and error to determine that lemon balm grows optimally in an MS medium supplemented with at least $50 \mathrm{~mm}$ sucrose (data not shown). To examine whether sucrose increases anthocyanin levels in lemon balm, we grew lemon balm plants in MS medium supplemented with $50,100,150,200,250$, or $300 \mathrm{~mm}$ sucrose for $20 \mathrm{~d}$ and measured the levels of anthocyanins in the leaves. As demonstrated in Figure 1, lemon balm leaves accumulated a high level of anthocyanins in response to sucrose in a dose-dependent manner: on treatment with $300 \mathrm{~mm}$ sucrose, the level of anthocyanins was increased up to 30 -fold compared with samples treated with $50 \mathrm{~mm}$ sucrose.

As shown in Figure 2A, lemon balm grew well in medium containing 150 or $200 \mathrm{~mm}$ sucrose. However, plants grown in $300 \mathrm{~mm}$ sucrose showed lower total dry weight as well as lower total fresh weight (Fig. 2B). Similar patterns were observed when we measured the leaf fresh weight and leaf dry weight of lemon balm plants in medium supplemented with 50,100,150, 200, 250, or $300 \mathrm{~mm}$ sucrose. Plant growth performance was severely damaged when the sucrose concentration was increased to 350 or $400 \mathrm{~mm}$ (Fig. S1; view supplemental figures online at http://hortsci.ashspublications.org). In our study, we observed that the relative chlorophyll content increased with sucrose treatment up to $200 \mathrm{~mm}$, after which it dropped sharply (Fig. 2C). However, we noticed that lemon balm leaves grown in high levels of sucrose became thicker compared with leaves grown in $50 \mathrm{~mm}$ sucrose. We therefore decided to determine the specific leaf weight (leaf weight per unit area) of the plants grown in different concentrations of sucrose and found a positive correlation between the level of sucrose treatment and the specific leaf weight (Fig. 2D).

$A$ high level of sucrose increases the overall flavonoid content in lemon balm. Because there are other types of flavonoids in plants besides anthocyanins, we wondered 


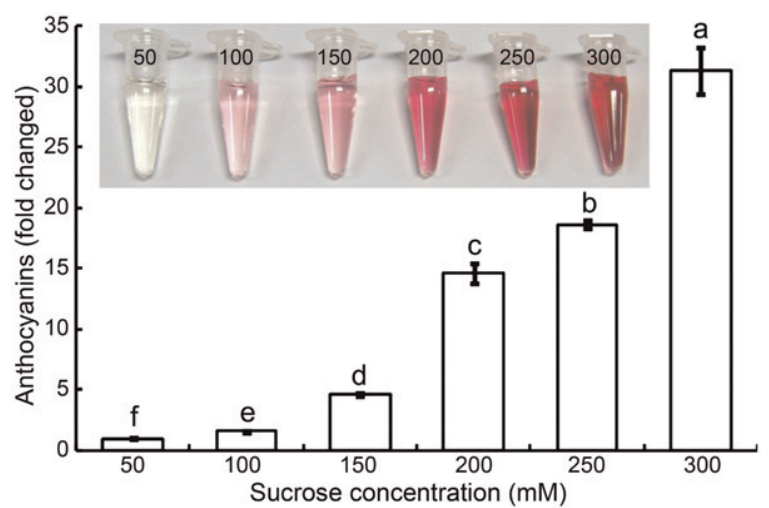

Fig. 1. Sucrose-induced accumulation of anthocyanins in lemon balm leaves. Plants were grown in full-strength Murashige and Skoog medium with 50,100, 150, 200, 250, or $300 \mathrm{~mm}$ sucrose and $0.7 \%$ agar for $20 \mathrm{~d}$ and anthocyanin levels were determined. Data represent the mean \pm SE from nine independent experimental replicates. The same letters indicate no significant difference at $P \leq 0.05$ (analysis of variance and Duncan's multiple range test). (Inset: Leaf extracts obtained for anthocyanin determination.)

A

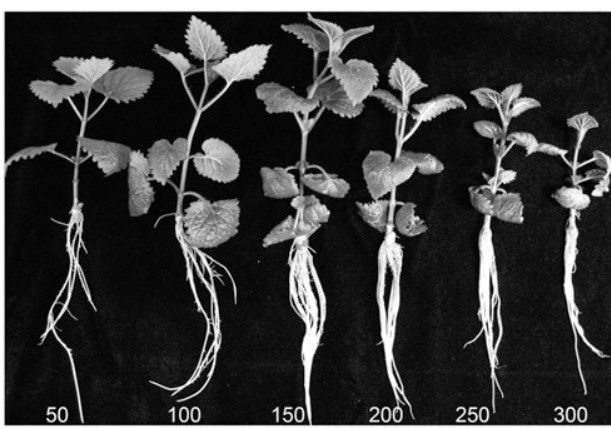

$\mathbf{B}$
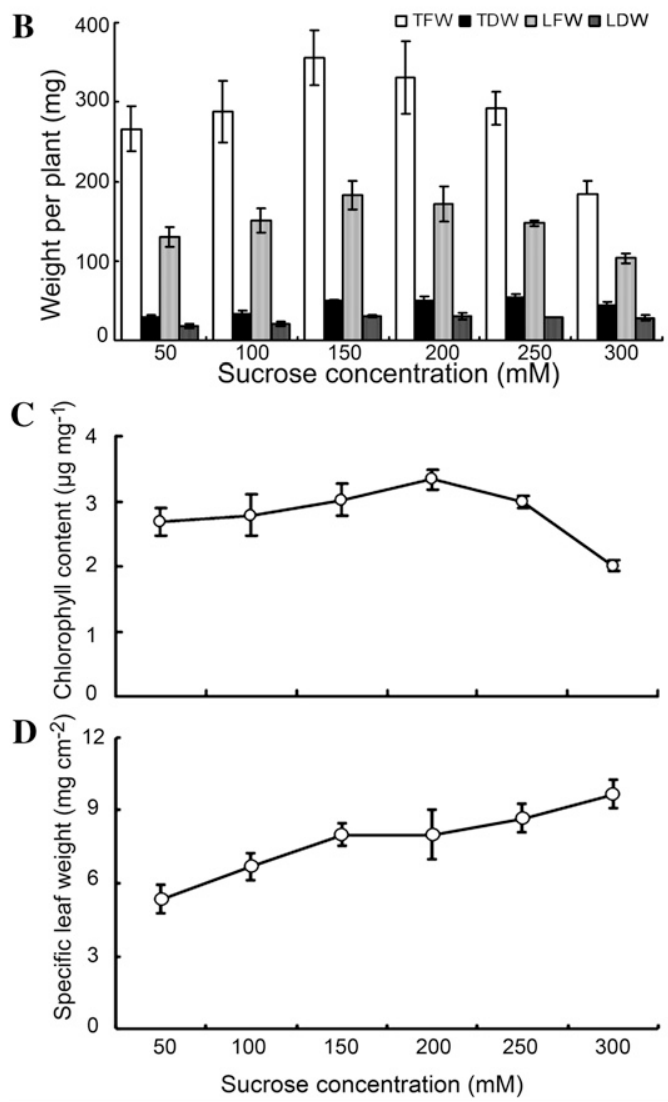

Fig. 2. Growth performance of lemon balm grown in Murashige and Skoog (MS) medium with various concentrations of sucrose. (A) Plants were grown in MS medium with 50, 100, 150, 200, 250, or $300 \mathrm{~mm}$ sucrose for 20 d. (B) Total fresh weight (TFW), total dry weight (TDW), leaf fresh weight (LFW), and leaf dry weight (LDW) per plant. (C) Chlorophyll content of the leaves. (D) Specific leaf weights measured as leaf weight per unit leaf area. Values are mean \pm SE from nine independent experimental replicates. if sucrose would also increase the total amount of flavonoids in lemon balm. Like anthocyanin, the highest flavonoid content [16.9 $\mu \mathrm{g}$ quercetin equivalents $\left.(\mathrm{QE}) \cdot \mathrm{mg}^{-1}\right]$ was recorded in leaves treated with $300 \mathrm{~mm}$ sucrose followed by $15.9 \mu \mathrm{g} \mathrm{QE} \cdot \mathrm{mg}^{-1}$ in the leaves treated with $250 \mathrm{~mm}$ sucrose. The lowest flavonoid content $\left(3.8 \mu \mathrm{g} \mathrm{QE} \cdot \mathrm{mg}^{-1}\right)$ was observed in plants grown in $50 \mathrm{~mm}$ sucrose (Fig. 3). The flavonoid content in plants treated with $300 \mathrm{~mm}$ sucrose was approximately fourfold higher than that in control plants. This result indicates that the overall flavonoid content can also be increased by sucrose treatment.

To confirm this result, we used liquid chromatography (LC)/MS to analyze the specific metabolites produced by plants treated with different sucrose concentrations. Among the various metabolites detected by LC/MS, those that showed significant differences in quantity among the plant groups were selected for PCA. A major separation between the lemon balm sample groups was easily achieved by combining the principal components $\mathrm{PC} 1$ and $\mathrm{PC} 2$, where the ellipse marked the $95 \%$ confidence level on the Hotelling T2 control chart. Because PC1 and PC2 accounted for $66.4 \%$ and $12.0 \%$ of the total variance, respectively, the majority of the variables ( $78.4 \%$ of the variance) were well described by the first two PCs. The PC values acquired from samples grown in different sucrose concentrations showed a clear counterclockwise trajectory on the score plot for PC1 and PC2, which followed the increments in sucrose concentration (Fig. 4A). On the loading plot corresponding to PC1 and PC2, the different metabolites corresponding to the scores were clearly distinguishable. In other words, the location of each sample in the score plot was affected by an identical placement of metabolites in the loading plot As shown in Figure 4B, metabolites with molecular weights (MWs) of 523.129 and 617.153 were more abundant than other metabolites in lemon balm grown in $300 \mathrm{~mm}$ sucrose. A metabolite with MW 615.714 was found at a higher concentration in lemon balm treated with $50 \mathrm{~mm}$ sucrose, whereas metabolites with MW 540.063 and 435.13 (presumptively delphinidin-3-arabinoside) were found to be more abundant in plants grown in $250 \mathrm{~mm}$ sucrose.

Based on the observation that the levels of anthocyanins in lemon balm leaves increased with increasing sucrose concentrations, we next assessed the reactive oxygen species (ROS) scavenging activities in the lemon balm leaves. In this assay, we observed that the DPPH scavenging activities in the leaf extracts varied from $27.3 \%$ to $73.9 \%$ depending on the concentration of sucrose tested (Table 1). The maximum scavenging activity $(73.9 \%)$ was obtained from leaves grown in $300 \mathrm{~mm}$ sucrose, whereas the lowest activity $(27.3 \%)$ was in leaves grown in $50 \mathrm{~mm}$ sucrose. As shown in Table 1, the $\mathrm{IC}_{50}$ values decreased significantly when plants were grown in 200,250 , or $300 \mathrm{~mm}$ sucrose. Lower $\mathrm{IC}_{50}$ values correlate with higher antioxidant 


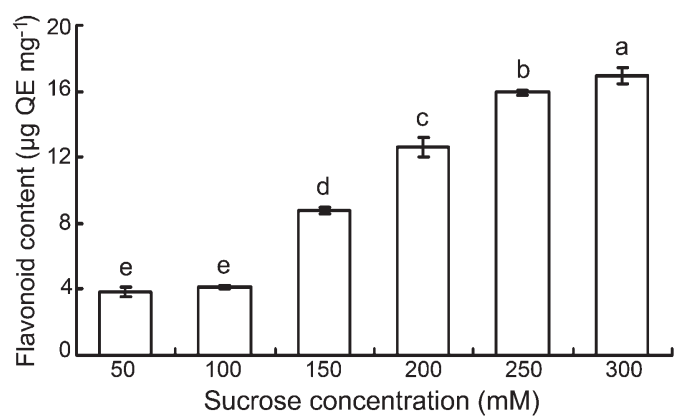

Fig. 3. Total flavonoid levels in lemon balm leaves increase following sucrose treatment in a dosedependent manner. Plants were grown in Murashige and Skoog medium supplemented with 50, 100, $150,200,250$, or $300 \mathrm{~mm}$ sucrose for $20 \mathrm{~d}$ and the total flavonoid concentration was determined according to established methods (Faudale et al., 2008). Values are means \pm SE from three independent experimental replicates. The same letters indicate no significant difference at $P \leq 0.05$ (analysis of variance and Duncan's multiple range test).

A
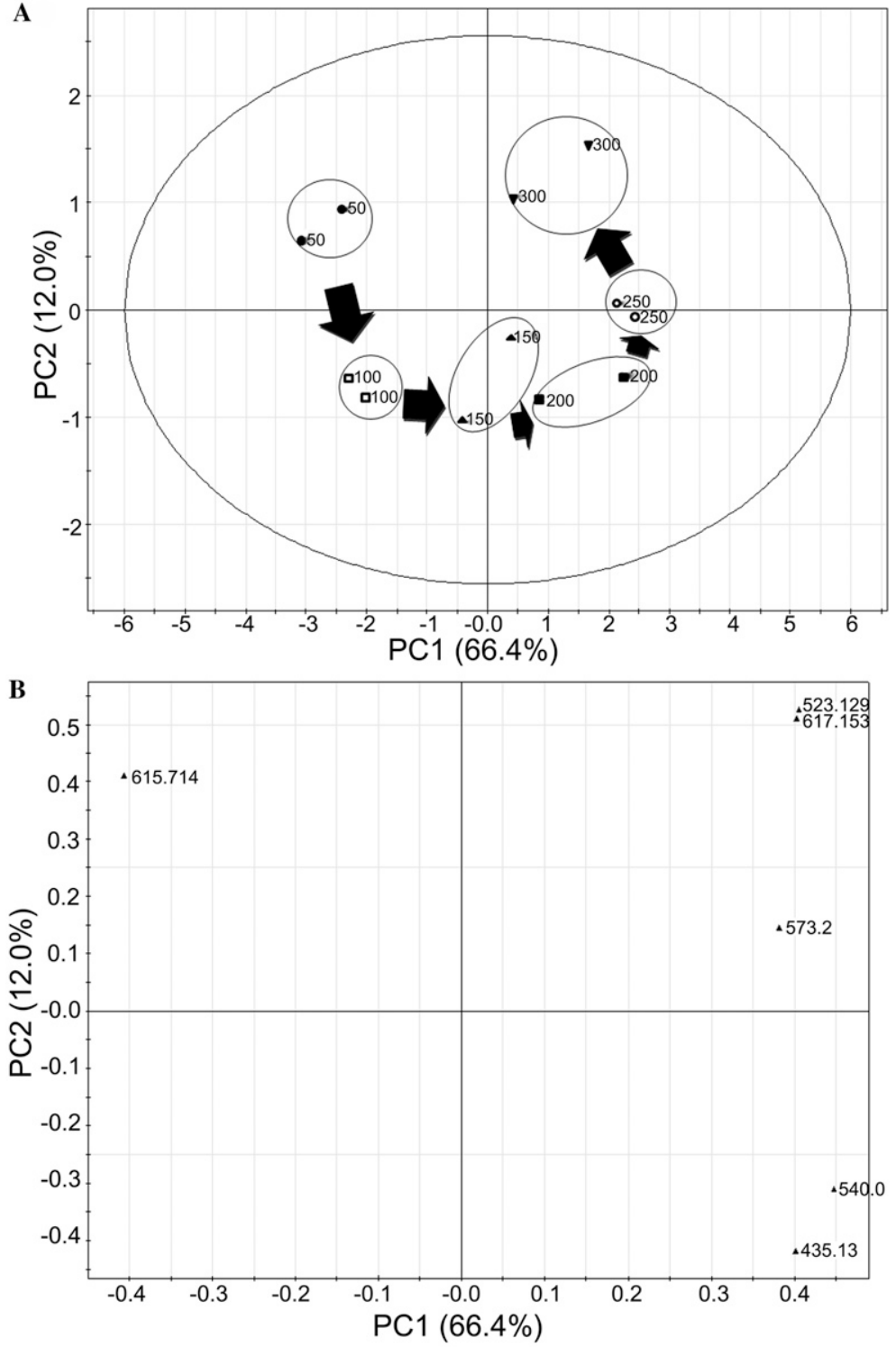

Fig. 4. Score plot of principal component analysis (PCA) of lemon balm extracts by combining PC1 and PC2. (A) Ellipse represents the Hotelling T2 with 95\% confidence. The labels 50, 100, 150, 200, 250, and 300 indicate lemon balm leaves grown in Murashige and Skoog media with 50, 100, 150, 200, 250, and $300 \mathrm{~mm}$ sucrose, respectively, for $20 \mathrm{~d}$. (B) The loading plot of the PCA for PC1 and PC2. PC1 has an explained variation of 0.67 and a predicted variation of 0.417 . activities in plant extracts (Patro et al., 2005) The lowest $\mathrm{IC}_{50}$ value $\left(52.9 \mu \mathrm{L} \cdot \mathrm{mL}^{-1}\right)$ was recorded from plants grown in $300 \mathrm{~mm}$ sucrose. The antioxidant activity of plants grown in $300 \mathrm{~mm}$ sucrose was 10 times higher than that in plants grown in $50 \mathrm{~mm}$ sucrose.

Effects of growth regulators on sucrosemediated anthocyanin accumulation. To examine the effects of phytohormones on sucrose-mediated anthocyanin accumulation in lemon balm, we altered the treatment conditions. A shorter period of incubation was used to avoid the degradation of hormone activity under long exposures to light. Instead of using plants grown directly in high sucrose medium for $20 \mathrm{~d}$, explants were allowed to grow in normal medium ( $50 \mathrm{~mm}$ sucrose) for $12 \mathrm{~d}$ and then were transferred to a liquid medium containing 50 or $300 \mathrm{~mm}$ sucrose. This medium was supplemented with one of five different growth regulators at concentrations of 0,5 , or $10 \mu \mathrm{M}$. The compounds tested were the ethylene precursor ACC and the hormones kinetin, ABA, IAA, and gibberellic acid (GA). After transfer to the liquid medium, the explants were incubated for a further $3 \mathrm{~d}$ before analysis. Figure 5 shows that treatments with ACC and ABA were able to enhance the anthocyanin accumulation significantly when combined with sucrose. However, GA negatively affected anthocyanin accumulation even at a high sucrose concentration (300 mM) (Figs. 5 and S2).

Next, we examined whether ABA or ethylene is involved in sucrose-mediated anthocyanin accumulation. Lemon balm plants were exposed for $3 \mathrm{~d}$ to 50 or $300 \mathrm{~mm}$ sucrose combined with the ethylene action inhibitor silver nitrate $\left(\mathrm{AgNO}_{3}\right)$, or with the $\mathrm{ABA}$ biosynthesis inhibitor fluridon, and anthocyanin levels were determined. The inhibitors were used at concentrations of $0,5,10$, or 20 $\mu \mathrm{M}$. The levels of anthocyanin decreased gradually in lemon balm leaves as the concentrations of fluridon or $\mathrm{AgNO}_{3}$ increased (Fig. 6). The results also indicate that $\mathrm{AgNO}_{3}$ is a more efficient inhibitor than fluridon in sucrose-mediated anthocyanin accumulation (Fig. 6). To examine whether these inhibitors also prohibit antioxidant activity in lemon balm grown at a high sucrose level, we measured the DPPH scavenging activities of leaves that had been exposed for $3 \mathrm{~d}$ to $0,5,10$, or $20 \mu \mathrm{M} \mathrm{AgNO}$ or fluridon in combination with $300 \mathrm{~mm}$ sucrose and observed increasing $\mathrm{IC}_{50}$ values with increased inhibitor concentrations (Table 2).

Because ROS are involved in osmotic stress, ROS production levels were assessed in lemon balm plants grown in 50, 100, 150, 200,250 , or $300 \mathrm{~mm}$ sucrose. As shown in Figure 7A, the production of ROS became increasingly evident in the leaves as the concentration of sucrose increased. We did not observe any significant ROS staining in leaves grown with up to $150 \mathrm{~mm}$ sucrose, and the leaves grown in $200 \mathrm{~mm}$ sucrose were only slightly stained. Because it was easy to detect ROS production with the $300 \mathrm{~mm}$ sucrose treatment, we confined our analysis 
Table 1. Antioxidant activities of lemon balm leaf extracts from plants grown in various concentrations of sucrose. ${ }^{\mathrm{z}}$

\begin{tabular}{|c|c|c|c|c|c|c|}
\hline \multicolumn{7}{|c|}{ Sucrose concentration } \\
\hline & $50 \mathrm{~mm}$ & $100 \mathrm{~mm}$ & $150 \mathrm{~mm}$ & $200 \mathrm{~mm}$ & $250 \mathrm{~mm}$ & $300 \mathrm{~mm}$ \\
\hline $1 \mathrm{C}_{50}\left(\mu \mathrm{L} \cdot \mathrm{mL}^{-1}\right)$ & $583.3 \pm 63.1 \mathrm{a}$ & $449.7 \pm 43.5 \mathrm{~b}$ & $229.9 \pm 12.0 \mathrm{c}$ & $60.5 \pm 1.6 \mathrm{~d}$ & $53.5 \pm 2.2 \mathrm{de}$ & $52.9 \pm 1.7 \mathrm{e}$ \\
\hline
\end{tabular}

to this condition for the remaining experiments. To determine the effects of various phytohormones on ROS production, plants were treated for $3 \mathrm{~d}$ with 50 or $300 \mathrm{~mm}$ sucrose combined with the ethylene precursor ACC or one of the four phytohormones, kinetin, ABA, IAA, and GA (each at 0,5 , or $10 \mu \mathrm{M})$. As shown in Figure 7B, dark ROS staining was evident in leaves grown in 50 mM sucrose and $10 \mu \mathrm{M}$ ABA. ROS staining intensified when plants were grown with 300 mM sucrose combined with 5 or $10 \mu \mathrm{M}$ ACC

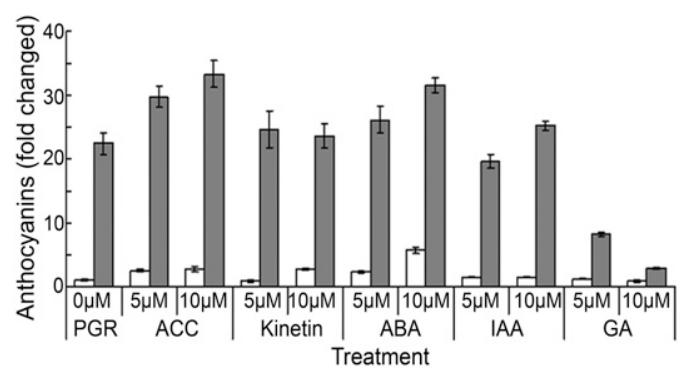

Fig. 5. The levels of anthocyanins in lemon balm plants grown in 50 or $300 \mathrm{~mm}$ sucrose in the presence of phytohormones. Plants grown in Murashige and Skoog medium with $50 \mathrm{~mm}$ sucrose for $12 \mathrm{~d}$ were transferred to liquid medium with $50 \mathrm{~mm}$ (open bar) or $300 \mathrm{~mm}$ (closed bar) sucrose in combination with 0,5 , or $10 \mu \mathrm{M}$ of the ethylene precursor 1-aminocyclopropane-carboxylic acid (ACC) or one of the phytohormones, kinetin, (+)-cis, transabscissic acid (ABA), indole-3-acetic acid (IAA), or gibberellic acid (GA). The plants were incubated for a further $3 \mathrm{~d}$ before anthocyanin measurements. Values are means \pm SE from nine independent experimental replicates. The same letters indicate no significant difference at $P \leq 0.05$ (analysis of variance and Duncan's multiple range test).

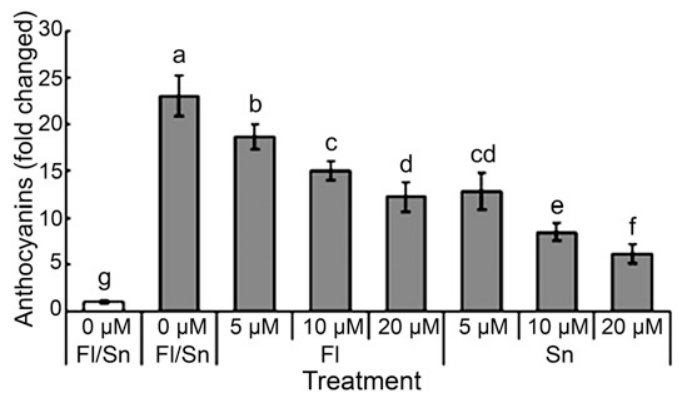

Fig. 6. The effect of fluridon and $\mathrm{AgNO}_{3}$ on anthocyanin accumulation in lemon balm leaves in the presence of $300 \mathrm{~mm}$ sucrose. Lemon balm plants grown in $50 \mathrm{~mm}$ sucrose for $12 \mathrm{~d}$ were incubated with $50 \mathrm{~mm}$ (open bar) or $300 \mathrm{~mm}$ (closed bar) sucrose together with fluridon (Fl) or $\mathrm{AgNO}_{3}(\mathrm{Sn})$ for $3 \mathrm{~d}$. Homogenized samples were analyzed using a microplate spectrophotometer to obtain anthocyanin contents. Values are means $\pm \mathrm{SE}$ from nine independent experimental replicates. The same letters indicate no significant difference at $P \leq 0.05$ (analysis of variance and Duncan's multiple range test).

or ABA. On the other hand, we observed that the ROS staining gradually decreased with increasing concentrations of GA, although sucrose was present at a concentration of 300 mM (Fig. 7B).

\section{Discussion}

Sugar-induced anthocyanin accumulation has been reported in various plant species (Nagira et al., 2006; Nagira and Ozeki, 2004; Solfanelli et al., 2006). One recent report suggests that strong anthocyanin induction can be triggered by sucrose but not by other sugars or by osmotic stress in Arabidopsis (Teng et al., 2005). However, the mechanisms controlling the induction of anthocyanin accumulation in lemon balm leaves in response to sucrose or to phytohormones remain unclear. In the experiments presented here, lemon balm leaves were exposed to high levels of sucrose, and in response, they accumulated anthocyanins as well as other flavonoids, which correlated with DPPH scavenging activity (Table 1). Moreover, delphinidin-3-arabinoside, which was found to be the primary anthocyanin by PCA analysis, was enriched in lemon balm grown in a high concentration of sucrose (Fig. 4B). Recently, Azuma and colleagues assessed the ROS scavenging activities of five purified anthocyanins using radical substrates such as the DPPH radical and the linoleic acid radical. The authors showed that delphinidin (delphinidin 3RGcaf5G) had a higher ROS scavenging activity than petunidin (petunidin 3RGc5G) (Azuma et al., 2008). Moreover, another recent report suggests that flavonoids can replace vitamin $\mathrm{E}$ as chain-breaking antioxidants in liver microsomal membranes (van Acker et al., 2000). This indicates that the delphinidin enriched in lemon balm leaves grown in $300 \mathrm{~mm}$ sucrose likely contributed to the enhanced ROS scavenging activity of the samples.

Table 2. DPPH (2, 2-diphenyl-1-picrylhydrazyl) scavenging activities of leaf extracts from plants treated with 50 or $300 \mathrm{~mm}$ sucrose and $0,5,10$, or $20 \mu \mathrm{M}$ fluridon or $\mathrm{AgNO}_{3}$ for $3 \mathrm{~d}^{\mathrm{z}}$

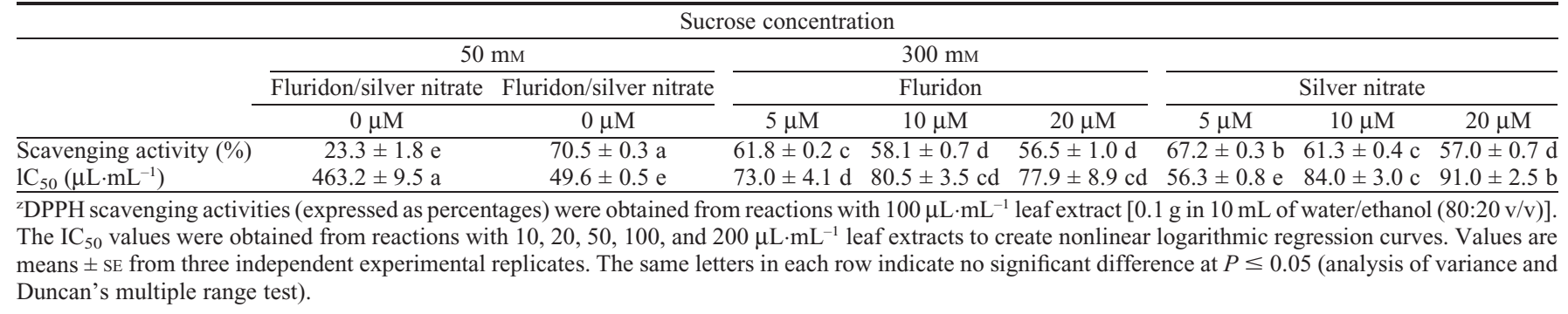


A

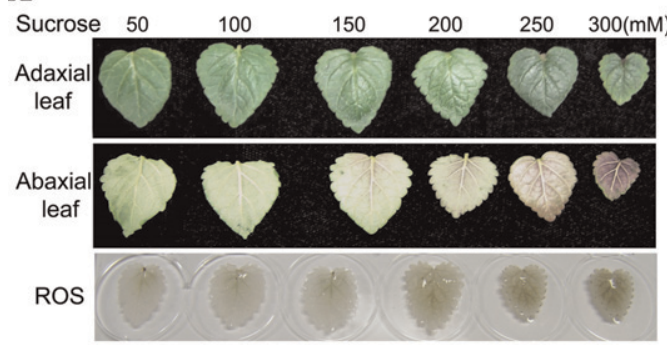

B

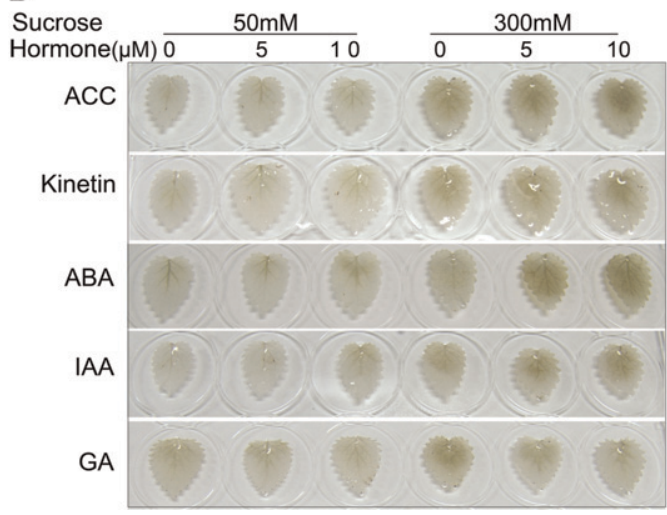

Fig. 7. Determination of reactive oxygen species (ROS) levels in lemon balm leaves grown in the presence of sucrose and phytohormones. Brown-colored staining obtained by the incubation of lemon balm leaves with 3, 3'-diaminobenzidine (DAB) was used as a marker for endogenous $\operatorname{ROS}\left(\mathrm{H}_{2} \mathrm{O}_{2}\right)$ production. (A) ROS staining of lemon balm leaves grown in various concentrations of sucrose for $20 \mathrm{~d}$. (B) Lemon balm leaves were treated with 0 , 5, or $10 \mu \mathrm{M} 1$-aminocyclopropane-carboxylic acid (ACC), kinetin, (+)-cis, transabscissic acid (ABA), indole-3-acetic acid (IAA), or gibberellic acid (GA) in the presence of 50 or $300 \mathrm{~mm}$ sucrose in liquid medium for $3 \mathrm{~d}$. Shown are the representative results from three independent experimental replicates.

We observed a positive synergistic effect on anthocyanin accumulation in lemon balm leaves when ABA or ACC were combined with sucrose in the growth medium. However, the phytohormones themselves slightly increased anthocyanin levels in plants grown in $50 \mathrm{~mm}$ sucrose (Fig. 5). When combined with $300 \mathrm{~mm}$ sucrose, ABA and ACC upregulated the accumulation of anthocyanins to similar extents. Consistent with this, the ABA biosynthesis inhibitor and the ethylene action inhibitor reduced the levels of sucroseinduced anthocyanin accumulation (Fig. 6). These results suggest positive roles of ABA and ethylene in the accumulation of anthocyanins in response to sucrose treatment. IAA also exhibited a positive effect on the accumulation of anthocyanins with sucrose treatment, but it did not reach the level triggered by $\mathrm{ABA}$ or $\mathrm{ACC}$. It seems that plants harbor different systems to trigger the accumulation of anthocyanins in response to hormones or sucrose. For example, in the Camptotheca acuminata cell culture system, anthocyanin production was significantly increased by the addition of kinetin (Pasqua et al., 2005), whereas we observed no significant effect with kinetin.

In our study, GA greatly reduced the accumulation of anthocyanins in a dosedependent manner (Fig. 5). Reductions in anthocyanin biosynthesis by GA have been reported in seedlings of tomato (Khan, 1980), radish (Jain and Guruprasad, 1989), and maize (White and Rivin, 2000). In another study, gibberellins were also shown to counteract the induction of anthocyanin biosynthesis by sucrose in Arabidopsis (Loreti et al., 2008). These authors demonstrated that $\mathrm{GA}_{3}$ repressed the induction of the $P A P 1$ and $P A P 2$ genes, which are responsive to sucrose in anthocyanin biosynthesis (Solfanelli et al., 2006; Teng et al., 2005). These reports suggest the existence of similar crosstalk mechanisms between sucrose and GA in the vegetative tissues of lemon balm and other species. In contrast, GA has been implicated as having a positive effect on the induction of flavonoid-specific genes, including $C H S$ and CHI, in flowers (Moalem-Beno et al., 1997; Neta-Sharir et al., 2000; Weiss et al., 1990). Therefore, different plant organs may harbor different signaling systems for mediating anthocyanin biosynthesis in response to phytohormones. It will be of interest to identify what components account for the variation in responses to phytohormones in different species and different plant parts.

ROS are regarded as toxic byproducts of aerobic metabolism that accumulate to a greater extent in cells under conditions of stress (Gratão et al., 2005, 2008). On the other hand, ROS have also been implicated as important second messenger molecules in the signaling and regulation of genes that are governed by various stimuli (Mittler, 2002). These contradictory roles are indicative of a complex modulation between divergent stress signals and ROS production for the integration of various cell signaling activities. In our study, the accumulation of anthocyanins was positively correlated with the level of ROS production (Fig. 7A). This clearly indicates that ROS may function as signaling molecules for the accumulation of anthocyanins in lemon balm plants. Both $\mathrm{ABA}$ and $\mathrm{ACC}$ increased ROS accumulation in lemon balm leaves grown with high levels of sucrose (Fig. 7B). ABA is also known to increase the activity of antioxidant enzymes, which can cause a decrease in oxidative stress. Thus, our results support the role of ABA in oxidative stress responses. We also observed that GA reduced the appearance of ROS in a dose-dependent manner, which was accompanied by a decrease in anthocyanin accumulation.

In conclusion, we have demonstrated for the first time that lemon balm plants respond to sucrose with increased levels of anthocyanins. Our studies revealed that sucrose significantly enhances the levels of flavonoids in lemon balm plants and that sucrose induction appears to be mediated by phytohormones, primarily $\mathrm{ABA}$ and ethylene. We identified delphinidin-3-arabinoside as the primary anthocyanin compound that accumulates in leaves grown in a highsucrose medium. Our results also showed that ROS levels are positively correlated with sucrose-mediated anthocyanin accumulation. These findings could be the foundation for future work in lemon balm at the molecular level.

\section{Literature Cited}

Azuma, K., A. Ohyama, K. Ippoushi, T. Ichiyanagi, A. Takeuchi, T. Saito, and H. Fukuoka. 2008 Structures and antioxidant activity of anthocyanins in many accessions of eggplant and its related species. J. Agr. Food Chem. 56:1015410159.

Brand-Williams, W., M.E. Cuvelier, and C. Berset. 1995. Use of a free radical method to evaluate antioxidant activity. Lebensm.-Wiss. Technol. 28:25-30.

Chen, Q.F., D. Liang-Ying, X. Shi, W. Yun-Sheng, L. Xiong-Lun, and W. Guo-Liang. 2007. The COII and DFR genes are essential for regulation of jasmonate-induced anthocyanin accumulation in Arabidopsis. J. Integr. Plant Biol. 49:1370-1377.

de Sousa, A.C., D.S. Alviano, A.F. Blank, P.B. Alves, C.S. Alviano, and C.R. Gattass. 2004. Melissa officinalis L. essential oil: Antitumoral and antioxidant activities. J. Pharm. Pharmacol. 56:677-681.

Devoto, A., C. Ellis, A. Magusin, H.S. Chang, C. Chilcott, T. Zhu, and J.G. Turner. 2005. Expression profiling reveals COII to be a key regulator of genes involved in wound- and methyl jasmonate-induced secondary metabolism, defense, and hormone interactions. Plant Mol. Biol. 58:497-513.

Faudale, M., F. Viladomat, J. Bastida, F. Poli, and C. Codina. 2008. Antioxidant activity and phenolic composition of wild, edible, and medicinal fennel from different Mediterranean countries. J. Agr. Food Chem. 56:1912-1920.

Fester, T. and G. Hause. 2005. Accumulation of reactive oxygen species in arbuscular mycorrhizal roots. Mycorrhiza 15:373-379. 
Galasinski, W., J. Chlabicz, A. Paszkiewicz-Gadek, C. Marcinkiewicz, and A. Gindzienski. 1996. The substances of plant origin that inhibit protein biosynthesis. Acta Pol. Pharm. 53: 311-318.

Geuenich, S., C. Goffinet, S. Venzke, S. Nolkemper, I. Baumann, P. Plinkert, J. Reichling, and O.T. Keppler. 2008. Aqueous extracts from peppermint, sage and lemon balm leaves display potent anti-HIV-1 activity by increasing the virion density. Retrovirology 5:27.

Gollop, R., S. Even, V. Colova-Tsolova, and A. Peri. 2002. Expression of the grape dihydroflavonol reductase gene and analysis of its promoter region. J. Expt. Bot. 53:1397-1409.

Gratão, P.L., C.C. Monteiro, L.E.P. Peres, and R.A. Azevedo. 2008. The isolation of antioxidant enzymes from mature tomato (cv. Micro-Tom) plants. HortScience 43:1608-1610.

Gratão, P.L., A. Polle, P.J. Lea, and R.A. Azevedo. 2005. Making the life of heavy metal-stress plants a little easier. Funct. Plant Biol. 32:481494.

Jain, V.K. and K.N. Guruprasad. 1989. Effect of chlorocholine chloride and gibberellic acid on the anthocyanin synthesis in radish seedlings. Physiol. Plant. 75:233-236.

Kennedy, D.O., W. Little, C.F. Haskell, and A.B. Scholey. 2006. Anxiolytic effects of a combination of Melissa officinalis and Valeriana officinalis during laboratory induced stress. Phytother. Res. 20:96-102.

Kennedy, D.O., A.B. Scholey, N.T. Tildesley, E.K. Perry, and K.A. Wesnes. 2004. Attenuation of laboratory-induced stress in humans after acute administration of Melissa officinalis (lemon balm). Psychosom. Med. 66:607-613.

Khan, M.I. 1980. Gibberellic acid bioassay based on the inhibition of anthocyanins production in tomato seedlings. Biol. Plant. 22:401-403.

Loreti, E., G. Povero, G. Novi, C. Solfanelli, A. Alpi, and P. Perata. 2008. Gibberellins, jasmonate and abscisic acid modulate the sucroseinduced expression of anthocyanin biosynthetic genes in Arabidopsis. New Phytol. 179: 1004-1016.

Ma, H.L., M.J. Qin, L.W. Qi, G. Wu, and P. Shu. 2007. Improved quality evaluation of Radix Salvia miltiorrhiza through simultaneous quan- tification of seven major active components by high-performance liquid chromatography and principal component analysis. Biomed. Chromatogr. 21:931-939.

Mittler, R. 2002. Oxidative stress, antioxidants and stress tolerance. Trends Plant Sci. 7:405410.

Moalem-Beno, D., G. Tamari, Y. Leitner-Dagan, A. Borochov, and D. Weiss. 1997. Sugardependent gibberellin-induced chalcone synthase gene expression in petunia corollas. Plant Physiol. 113:419-424.

Muller, S.F. and S. Klement. 2006. A combination of valerian and lemon balm is effective in the treatment of restlessness and dyssomnia in children. Phytomedicine 13:383-387.

Nagira, Y., K. Ikegami, T. Koshiba, and Y. Ozeki. 2006. Effect of ABA upon anthocyanin synthesis in regenerated torenia shoots. J. Plant Res. 119:137-144.

Nagira, Y. and Y. Ozeki. 2004. A system in which anthocyanin synthesis is induced in regenerated torenia shoots. J. Plant Res. 117:377-383.

Neff, M.M. and J. Chory. 1998. Genetic interactions between phytochrome A, phytochrome B, and cryptochrome 1 during Arabidopsis development. Plant Physiol. 118:27-35.

Neta-Sharir, I., O. Shoseyov, and D. Weiss. 2000 Sugars enhance the expression of gibberellininduced genes in developing petunia flowers. Physiol. Plant. 109:196-202.

Pasqua, G., B. Monacellia, N. Mulinacci, S. Rinaldi, C. Giaccherini, M. Innocenti, and F. Vinceri. 2005. The effect of growth regulators and sucrose on anthocyanin production in Camptotheca acuminata cell cultures. Plant Physiol. Biochem. 43:293-298.

Patro, B.S., A.K. Bauri, S. Mishra, and S. Chattopadhyay. 2005. Antioxidant activity of Myristica malabarica extracts and their constituents. J. Agr. Food Chem. 53:6912-6918.

Porra, R.J., W.A. Thompson, and P.A. Kriedmann. 1989. Determination of accurate extinction coefficients and simultaneous equations for assaying chlorophylls $a$ and $b$ extracted with four different solvents: Verification of the concentration of chlorophyll standards by atomic absorption spectroscopy. Biochim. Biophys. Acta 975:384-394.
Rolland, F., B. Moore, and J. Sheen. 2002. Sugar sensing and signaling in plants. Plant Cell 14:S185-S205.

Rook, F. and M.W. Bevan. 2003. Genetic approaches to understanding sugar response pathways. J. Expt. Bot. 54:495-501.

Smeekens, S. 2000. Sugar-induced signal transduction in plants. Annu. Rev. Plant Physiol. Plant Mol. Biol. 51:49-81.

Solfanelli, C., A. Poggi, E. Loreti, A. Alpi, and P. Perata. 2006. Sucrose-specific induction of the anthocyanin biosynthetic pathway in Arabidopsis. Plant Physiol. 140:637-646.

Teng, S., J. Keurentjes, L. Bentsink, M. Koornneef, and S. Smeekens. 2005. Sucrose-specific induction of anthocyanin biosynthesis in Arabidopsis requires the MYB75/PAP1 gene. Plant Physiol. 139:1840-1852.

Tolstikov, V.V., O. Fiehn, and N. Tanaka. 2007. Application of liquid chromatography-mass spectrometry analysis in metabolomics, p. 141-155. In: Weckwerth, W. (ed.). Metabolomics methods and protocols. Humana Press, Totowa, NJ.

Tonelli, C., E. Cominelli, D. Allegra, and M. Galbiati. 2007. Plant tolerance to drought and salinity: Modulation of transcription factors. Proc. 18th International Conference on Arabidopsis Research. p. 176.

van Acker, F.A., O. Schouten, G.R. Haenen, W.J. van der Vijgh, and A. Bast. 2000. Flavonoids can replace alpha-tocopherol as an antioxidant. FEBS Lett. 473:145-148.

Weiss, D., A.J.V. Tunen, A.H. Halevy, J.N.M. Mol, and A.G.M. Gerats. 1990. Stamens and gibberellic acid in the regulation of flavonoid gene expression in the corolla of Petunia hybrida. Plant Physiol. 94:511-515.

White, C.N. and C.J. Rivin. 2000. Gibberellins and seed development in maize. II. Gibberellin synthesis inhibition enhances abscisic acid signaling in cultured embryos. Plant Physiol. 122:1089-1097.

Yamasaki, K., M. Nakano, T. Kawahata, H. Mori, T. Otake, N. Ueba, I. Oishi, R. Inami, M. Yamane, M. Nakamura, H. Murata, and T. Nakanishi. 1998. Anti-HIV-1 activity of herbs in Labiatae. Biol. Pharm. Bull. 21:829833 . 

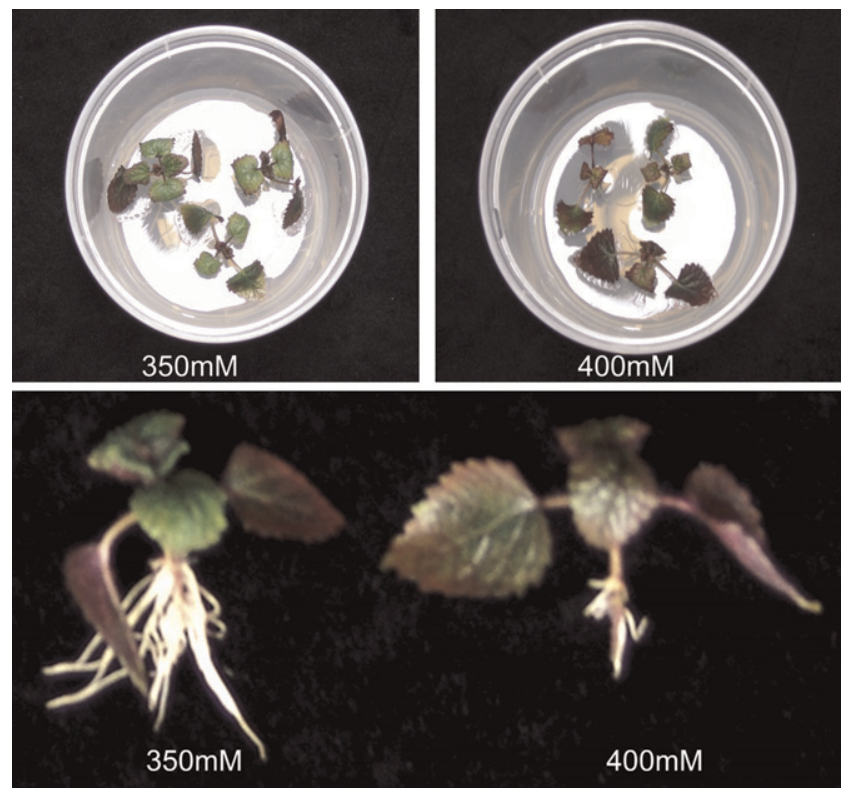

Fig. S1 Inhibition of plant growth by high levels of sucrose. Plants were grown in Murashige and Skoog medium supplemented with 350 or $400 \mathrm{~mm}$ sucrose for $20 \mathrm{~d}$ before the photograph was taken. Shown are the representative results from three independent experimental replicates.

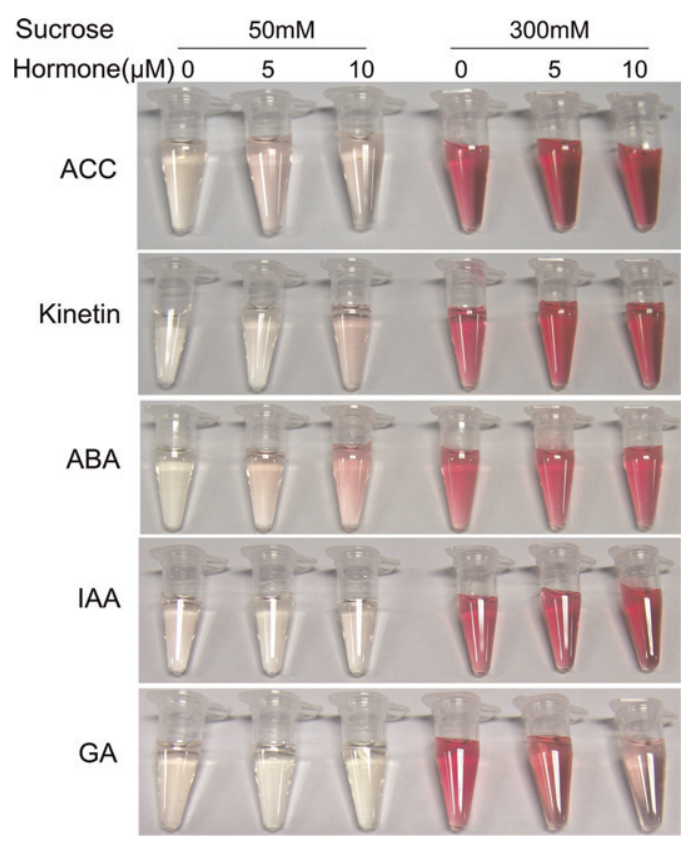

Fig. S2 Pigments of anthocyanins extracted from lemon balm leaves treated for $3 \mathrm{~d}$ with 0,5 , or $10 \mu \mathrm{M} 1-$ aminocyclopropane-carboxylic acid (ACC), kinetin, (+)-cis, transabscissic acid (ABA), indole-3acetic acid (IAA), or gibberellic acid (GA) along with $50 \mathrm{~mm}$ or $300 \mathrm{~mm}$ sucrose in a liquid medium. Shown are the representative results from three independent experimental replicates. 